\title{
Innovative Materials for Energy Efficient Buildings
}

\author{
Laurent Pilon \\ University of California, Los Angeles, USA \\ ${ }^{1}$ Mechanical and Aerospace Engineering Dept. \\ ${ }^{2}$ Institute of the Environment and Sustainability \\ ${ }^{3}$ California Nanosystems Institute \\ www.seas.ucla.edu/ pilon/
}

Building operations account for about $40 \%$ of total US primary energy resource consumption. Approximately 30-40\% of this energy is consumed for heating, ventilation, and air conditioning (HVAC). In addition, direct greenhouse gas (GHG) emissions from buildings accounts for approximately $12 \%$ of the total GHG emission in the U.S., mainly due to combustion of fossil fuels for heating in the winter months. The easiest way to reduce building energy consumption and the associated GHG emissions is to reduce heat losses through the building envelope. Moreover, windows are arguably the weakest constituent of the building envelope. While double pane windows have excellent thermal, optical, and acoustic performance, their adoption has been relatively slow. In fact, between 30 and $40 \%$ of single pane windows remain in use in the Northeastern/Midwestern and Southern regions of the US, respectively. This presentation will describe the synthesis and characterization of novel building materials and their impact on building energy consumptions.

First, a common strategy to improve building energy efficiency is to use materials with a large thermal mass such as concrete or brick. While these materials can store large amounts of energy per unit mass, they operate passively. Here, we will discuss embedding phase change materials (PCMs) in cementitious composite building materials to add an active dimension to the thermal behavior of building envelope. Indeed, by reversibly undergoing melting and freezing during the course of a day, PCMs are able to actively and adaptively absorb and release their latent heat. This, in turns, reduces and delays the thermal load on the building and reduces the cost for HVAC operations.

Second, we will present new synthesis methods and characterization of both thermally insulating and optically transparent mesoporous silica monoliths. The thermal conductivity of transparent materials (e.g., glass) can be reduced by introducing porosity. However, it is typically accompanied by the presence of large pores which scatter visible light and render the material opaque or translucent instead of transparent. Here, we present thick mesoporous silica monoliths synthesized at room temperature and atmospheric pressure and featuring porosity up to $90 \%$ with narrow pore size distribution with all pores less than $20 \mathrm{~nm}$ in diameter resulting in excellent optical clarity and very low thermal conductivity. Finally, the thermal performance of the windows solution integrating these new materials will be discussed in terms of U-value and solar heat gain coefficient. 